\title{
Polo-like kinase 4 as a potential predictive biomarker of chemoradioresistance in locally advanced rectal cancer
}

\author{
Hyunseung $\mathrm{Oh}^{1}$, Soon Gu Kim², Sung Uk Bae ${ }^{3}$, Sang Jun Byun ${ }^{4}$, Shin Kim5, \\ Jae-Ho Lee ${ }^{6}$, Ilseon Hwang ${ }^{1}$, Sun Young Kwon ${ }^{1}$, Hye Won Lee ${ }^{1}$ \\ Departments of ${ }^{1}$ Pathology and ${ }^{2}$ Education Support Center, ${ }^{3}$ Division of Colorectal Surgery, Department of Surgery, \\ Departments of ${ }^{4}$ Radiation Oncology, ${ }^{5}$ mmunology, and ${ }^{6}$ Anatomy, Keimyung University School of Medicine, Daegu, Korea
}

\begin{abstract}
Background: Polo-like kinase 4 (PLK4) is a serine/threonine protein kinase located in the centriole of the chromosome during the cell cycle. PLK4 overexpression has been described in a variety of many common human epithelial tumors. Conversely, PLK4 acts as a haploinsufficient tumor suppressor in some situations, highlighting the importance of strict regulation of PLK4 expression, activity, and function. Meanwhile, the importance of chemoradiation resistance in rectal cancer is being emphasized more than ever. We aimed to analyze PLK4 expression and the tumor regression grade (TRG) in patients with rectal cancer, treated with chemoradiotherapy (CRT). Methods: A retrospective study was conducted on 102 patients with rectal cancer who received preoperative CRT. Immunohistochemistry for PLK4 in paraffin-embedded tissue was performed from the biopsy and surgical specimens. Results: We found significant association between high expression of PLK4 and poor response to neoadjuvant CRT (according to both Mandard and The Korean Society of Pathologists TRG systems) in the pre-CRT specimens. Other clinicopathologic parameters did not reveal any correlation with PLK4 expression. Conclusions: This study revealed an association between high expression of PLK4 in the pre-CRT specimens and TRG. Our results indicated that PLK4 could potentially be a new predictor for CRT effect in patients with rectal cancer.
\end{abstract}

Key Words: Polo-like kinase 4; Rectal neoplasms; Chemoradiotherapy; Biomarker

Received: August 5, 2021 Revised: September 29, 2021 Accepted: October 7, 2021

Corresponding Author: Hye Won Lee, MD, PhD, Department of Pathology, Keimyung University School of Medicine, 1095 Dalgubeol-daero, Dalseo-gu, Daegu 42601, Korea Tel: +82-53-258-4260, Fax: +82-53-258-7382, E-mail: hwlee@dsmc.or.kr

The 5-year survival rate for rectal cancer patients undergoing surgery is $50 \%$. This is because even with curative resection, the risk of local recurrence or distant metastasis is high [1]. Therefore, improving survival remains an important issue, even in resectable rectal cancer. Meanwhile, in patients with rectal cancer, preoperative chemoradiotherapy (CRT) significantly improves the overall and cancer-specific survival rates compared to surgery alone. Therefore, chemoradioresistance is an important challenge in rectal cancer and a major aspect of its treatment.

Polo-like kinase 4 (PLK4) is a serine/threonine kinase located in the centriole of the chromosome throughout the cell cycle and is essential for centriole duplication [2-5]. PLK4 expression has been described in a variety of human solid tumors [6]. In particular, high levels of PLK4 mRNA have been detected in triplenegative breast cancers, which are resistant to conventional systemic therapy [7]. PLK4 expression upregulation independently induces aneuploidy, loss of cell polarity, and hyperplasia in certain non-transformed cell lines and proliferative tissues [8,9]. In the context of p53 dysfunction, elevated PLK4 expression contributes to aneuploidy and tumorigenesis $[9,10]$. Contrarily, PLK4 acts as a haploinsufficient tumor suppressor in some situations. Rosario et al. pointed out that haploid levels of PLK 4 interfered with RhoGTPase function during cytokinesis, leading to aneuploidy and tumorigenesis, thus suggesting that early loss of heterozygosity in PLK4 is one of the factors leading to hepatocellular carcinogenesis $[11,12]$. These results underscore the importance of strict regulation of PLK4 expression, activity, and function [13]. However, studies on PLK4 expression in patients with rectal cancer are limited. Thus, this study aimed to retrospectively analyze PLK4 expression in patients with rectal carcinoma treated with preoperative CRT in both preoperative biopsy specimens and postoperative surgical specimens. 


\section{MATERIALS AND METHODS}

\section{Patients and sample collection}

The electronic records of rectal cancer patients treated at Keimyung University Dongsan Hospital were reviewed retrospectively. Among them, 102 cases containing both biopsy and surgical specimens were chosen. These tissue specimens along with patient clinical data (age, sex, date of surgery, recurrence, death, data on time to recurrence or death) and records of clinicopathological features (differentiation, lymphovascular invasion, $\mathrm{pT}$ category, $\mathrm{pN}$ category, $\mathrm{pM}$ category, microsatellite instability [MSI] status, KRAS, NRAS and BRAF mutations) of the rectal cancer were collected.

Among the various grading systems devised to evaluate chemoradioresistance, the Mandard system [14] was used (MandardTRG [tumor regression grade]). Additionally, the KSP-TRG system, newly proposed by the Gastrointestinal Pathology Research Group of the Korean Society of Pathologists (KSP), based on the 8th edition of American Joint Committee on Cancer and the College of American Pathologists cancer protocol, was also used and data collected [15]. This grade is divided into four tiers: grade 0 (no viable cancer cells, complete response), grade 1 (single cells or rare small groups of cancer cells, near-complete response), grade 2 (residual cancer with evident tumor regression, but more than single cells or rare small groups of cancer cells, partial response), grade 3 (extensive residual cancer with no evident tumor regression, poor or no response).
Overall survival (OS) was defined as the duration from the date of surgery to the date of the last follow-up visit or the date of death due to any cause, whereas disease-free survival (DFS) was defined as the duration from surgery to any type of recurrence.

\section{Immunohistochemistry}

We selected formalin-fixed paraffin-embedded (FFPE) tissue samples from rectal cancer tissues and used them for tissue microarray (TMA) construction. Of the 102 patients with rectal cancer, tissue specimens from both before CRT (biopsy specimen) and after CRT (surgery specimen) were selected for 71 patients. Only biopsy specimens were selected from 31 patients with no or few cells remaining in the surgical specimens. Representative tumor areas were identified by two pathologists (H.O. and H.W.L.) in hematoxylin and eosin-stained tissue sections. After the pathologists' review, TMAs were assembled from triplicate $5 \mathrm{~mm}$ cores of the FFPE tumor samples. Immunohistochemical staining was performed on the TMA sections with an antibody against PLK4 (12952-1-AP, 1:400, ProteinTech Group, Inc., Chicago, IL, USA) using the Ventana BenchMark XT Automated System following the manufacturer's protocol (Ventana Medical Systems, Tucson, AZ, USA).

\section{Evaluation of immunohistochemistry}

The histoscore (H-score) method was used to determine the immunohistochemical expression of PLK4. The H-score was calculated by multiplying the intensity score by the percentage
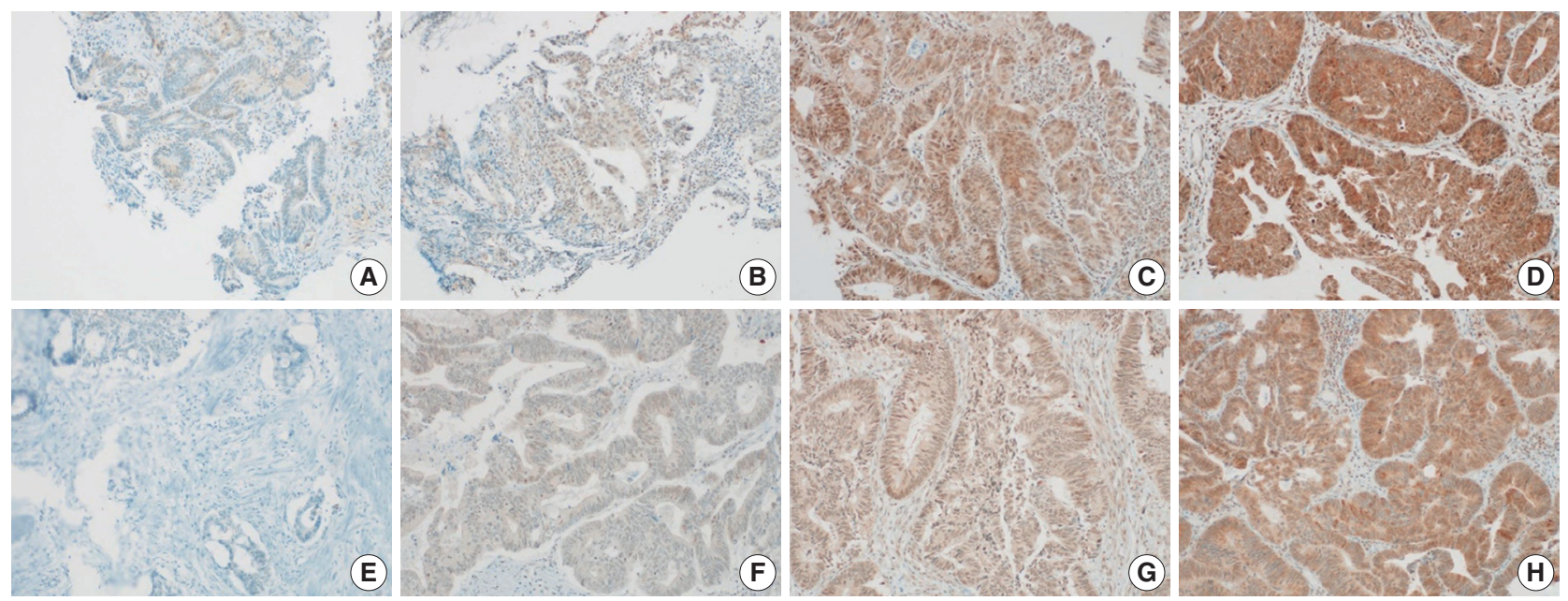

Fig. 1. Immunohistochemistry analyses of PLK4 expression in rectal cancer tissues. The expression of PLK is evaluated by $\mathrm{H}$-score; intensity multiplied by percentage of positive cells. (A) Score 0 (negative staining) of PLK4 expression of pre-CRT specimen. (B) Score 1 (weak staining) of PLK4 expression of pre-CRT specimen. (C) Score 2 (medium staining) of PLK4 expression of pre-CRT specimen. (D) Score 3 (strong staining) of PLK4 expression of pre-CRT specimen. (E) Score 0 (negative staining) of PLK4 expression of post-CRT specimen. (F) Score 1 (weak staining) of PLK4 expression of post-CRT specimen. (G) Score 2 (medium staining) of PLK4 expression of post-CRT specimen. (H) Score 3 (strong staining) of PLK4 expression of post-CRT specimen. PLK4, Polo-like kinase 4; CRT, chemoradiotherapy. 
of positive cells and ranges from 0 to 300 points [16]. We scored both the nucleus and cytoplasm of previously selected samples. The intensities were scored as "0" (negative staining), "1" (weak staining), "2" (medium staining), and "3" (strong staining) (Fig. 1). Based on the median H-score, two groups were formed for statistical analysis: "high expression" (H-score > median) and "low expression" (H-score $\leq$ median).

\section{Statistical analysis}

All analyses were performed using IBM SPSS Statistics software ver. 26.0 (IBM Corp., Armonk, NY, USA). Chi-square with Fischer's exact tests analysis were performed. Kaplan-Meier curves were used for survival analysis. Ordinal logistic regression model was used to compare the influence of individual factors, including age, sex, $\mathrm{pT}$ category, $\mathrm{pN}$ category, MSI status, and nuclear and cytoplasmic PLK4 expression of the pre-CRT specimen, on the KSP-TRG system. A two-tailed p-value of less than .05 indicated statistical significance.

\section{RESULTS}

\section{Cohort characteristics}

The median age of the cohort was 65 years (range, 32 to 85 years). Among the 102 patients, 70 (69\%) were men and 32 (31\%) were women. Rectal cancer recurred in 28 patients (27\%), and 11 patients (11\%) died. All characteristics of the 102 patients with rectal cancer are shown in Table 1.

\section{PLK4 expression and TRG}

High nuclear PLK4 expression in pre-CRT specimens (H-score $>160)$ correlated with high Mandard-TRG $(\mathrm{p}<.001)$ and KSPTRG $(\mathrm{p}<.001)$. The Mandard-TRG $(\mathrm{p}<.001)$ and KSP-TRG $(\mathrm{p}<.001)$ also showed a significant correlation in the group. Furthermore, high cytoplasmic PLK4 expression in the pre-CRT specimens (H-score > 155) correlated with high Mandard-TRG $(\mathrm{p}=.022)$ and KSP $(\mathrm{p}=.013)$ systems. Again, the Mandard-TRG $(\mathrm{p}=.002)$ and KSP-TRG $(\mathrm{p}=.004)$ were also significantly correlated within groups. There was no association between PLK4 expression in the pre-CRT specimens and other parameters. The results mentioned above are summarized in Table 2. Nuclear PLK4 expression in the post-CRT specimens was correlated with sex $(p=.015)$ and mortality $(\mathrm{p}=.035)$. There was no association between PLK4 expression in the post-CRT specimens and other parameters. The results mentioned above are summarized in Table 3.

High Mandard-TRG correlated with high pT category $(\mathrm{p}<$
Table 1. Clinicopathological parameters in 102 cases of rectal cancer patients

\begin{tabular}{|c|c|}
\hline Characteristic & No. (\%) \\
\hline \multicolumn{2}{|l|}{ Age (yr) } \\
\hline$<65$ & $50(49.0)$ \\
\hline$\geq 65$ & $52(51.0)$ \\
\hline Median (range) & $65(32-85)$ \\
\hline \multicolumn{2}{|l|}{ Sex } \\
\hline Male & $70(68.6)$ \\
\hline Female & $32(31.4)$ \\
\hline \multicolumn{2}{|l|}{ Differentiation } \\
\hline Well & $5(4.9)$ \\
\hline Moderate & $89(87.3)$ \\
\hline Poorly & $5(4.9)$ \\
\hline \multicolumn{2}{|c|}{ Lymphovascular invasion } \\
\hline Absent & $71(69.6)$ \\
\hline Present & $23(22.5)$ \\
\hline \multicolumn{2}{|l|}{ pT category } \\
\hline $\mathrm{Tx}-\mathrm{T} 2$ & $50(49.0)$ \\
\hline T3-T4 & $52(51.0)$ \\
\hline \multicolumn{2}{|l|}{ pN category } \\
\hline NO & $71(69.6)$ \\
\hline $\mathrm{N} 1-\mathrm{N} 2$ & $31(30.4)$ \\
\hline \multicolumn{2}{|l|}{ pM category } \\
\hline MO & $101(99.0)$ \\
\hline M1 & $1(1.0)$ \\
\hline \multicolumn{2}{|c|}{ TRG of Mandard (group) } \\
\hline TRG1, 2 & $27(26.5)$ \\
\hline TRG3 & $37(36.3)$ \\
\hline TRG4, 5 & $38(37.3)$ \\
\hline \multicolumn{2}{|l|}{ TRG of KSP (group) } \\
\hline TRGO, 1 & $27(26.5)$ \\
\hline TRG2, 3 & $75(73.5)$ \\
\hline \multicolumn{2}{|l|}{ TRG of Mandard } \\
\hline TRG1 & $16(15.7)$ \\
\hline TRG2 & $11(10.8)$ \\
\hline TRG3 & $37(36.3)$ \\
\hline TRG4 & $28(27.5)$ \\
\hline TRG5 & $10(9.8)$ \\
\hline \multicolumn{2}{|l|}{ TRG of KSP } \\
\hline TRGO & $16(15.7)$ \\
\hline TRG1 & $11(10.8)$ \\
\hline TRG2 & $39(38.2)$ \\
\hline TRG3 & $36(35.3)$ \\
\hline \multicolumn{2}{|l|}{ MSI } \\
\hline MSS and MSI-L & $93(91.2)$ \\
\hline MSI-H & $9(8.8)$ \\
\hline \multicolumn{2}{|l|}{ Recurrence } \\
\hline No & $74(72.5)$ \\
\hline Yes & $28(27.5)$ \\
\hline \multicolumn{2}{|l|}{ Death } \\
\hline No & 91 (89.2) \\
\hline Yes & $11(10.8)$ \\
\hline
\end{tabular}

TRG, tumor regression grade; KSP, The Korean Society for Pathologists; MSI, microsatellite instability; MSS, microsatellite stable; MSI-L, MSI-low; MSI-H, MSI-high. 
Table 2. Correlation of PLK4 expression of pre-CRT specimen with clinicopathological parameters in 102 cases of rectal cancer patients

\begin{tabular}{|c|c|c|c|c|c|c|}
\hline & \multicolumn{3}{|c|}{ Nuclear stain of PLK4 in pre-CRT specimen } & \multicolumn{3}{|c|}{ Cytoplasmic stain of PLK4 in pre-CRT specimen } \\
\hline & $\begin{array}{l}\text { Low expression } \\
(H \text {-score } \leq 160)\end{array}$ & $\begin{array}{c}\text { High expression } \\
(H \text {-score }>160)\end{array}$ & p-value & $\begin{array}{l}\text { Low expression } \\
(\text { H-score } \leq 155)\end{array}$ & $\begin{array}{l}\text { High expression } \\
(\text { H-score }>155)\end{array}$ & $p$-value \\
\hline Age (yr) & & & .196 & & & $>.99$ \\
\hline$<65$ & $18(20.9)$ & $24(27.9)$ & & $21(24.4)$ & $21(24.4)$ & \\
\hline$\geq 65$ & $25(29.1)$ & $19(22.1)$ & & $22(25.6)$ & $22(25.6)$ & \\
\hline Sex & & & .486 & & & .816 \\
\hline Male & $31(36.0)$ & $28(32.6)$ & & $29(33.7)$ & $30(34.9)$ & \\
\hline Female & $12(14.0)$ & $15(17.4)$ & & $14(16.3)$ & $13(15.1)$ & \\
\hline Differentiation & & & .999 & & & .999 \\
\hline Well & $2(2.4)$ & $2(2.4)$ & & $2(2.4)$ & $2(2.4)$ & \\
\hline Moderate & $39(45.9)$ & $38(44.7)$ & & $39(45.9)$ & $38(44.7)$ & \\
\hline Poorly & $2(2.4)$ & $2(2.4)$ & & $2(2.4)$ & $2(2.4)$ & \\
\hline Lymphovascular invasion & & & .118 & & & .150 \\
\hline Absent & $26(32.5)$ & $35(43.8)$ & & 27 (33.8) & $34(42.5)$ & \\
\hline Present & $12(15.0)$ & $7(8.8)$ & & $12(15.0)$ & $7(8.8)$ & \\
\hline pT category & & & .518 & & & .518 \\
\hline $\mathrm{Tx}-\mathrm{T} 2$ & $23(26.7)$ & $20(23.3)$ & & $20(23.3)$ & $23(26.7)$ & \\
\hline T3-T4 & $20(23.3)$ & $23(26.7)$ & & $23(26.7)$ & $20(23.3)$ & \\
\hline pN category & & & .323 & & & .138 \\
\hline NO & $30(34.9)$ & $34(39.5)$ & & $29(33.7)$ & $35(40.7)$ & \\
\hline $\mathrm{N} 1-\mathrm{N} 2$ & $13(15.1)$ & $9(10.5)$ & & $14(16.3)$ & $8(9.3)$ & \\
\hline pM category & & & $>.99$ & & & .309 \\
\hline MO & $42(49.4)$ & $42(49.4)$ & & 41 (48.2) & $43(50.5)$ & \\
\hline M1 & $1(1.2)$ & 0 & & $1(1.2)$ & 0 & \\
\hline TRG of Mandard & & & $<.001$ & & & .022 \\
\hline TRG1, 2 & $19(22.1)$ & $3(3.5)$ & & $16(18.6)$ & $6(7.0)$ & \\
\hline TRG3 & $13(15.1)$ & $19(22.1)$ & & $16(18.6)$ & $16(18.6)$ & \\
\hline TRG4, 5 & $11(12.8)$ & $21(24.4)$ & & $11(12.8)$ & $21(24.4)$ & \\
\hline TRG of KSP & & & $<.001$ & & & .013 \\
\hline TRG0, 1 & $19(22.1)$ & $3(3.5)$ & & $16(18.6)$ & $6(7.0)$ & \\
\hline TRG2, 3 & $24(27.9)$ & $40(46.5)$ & & 27 (31.4) & $37(43.0)$ & \\
\hline MSI & & & $>.99$ & & & $>.99$ \\
\hline MSS and MSI-L & $39(45.3)$ & 38 (44.2) & & 37 (52.1) & $28(39.4)$ & \\
\hline MSI-H & $4(4.7)$ & $5(5.8)$ & & $4(5.6)$ & $2(2.8)$ & \\
\hline Recurrence & & & .952 & & & .357 \\
\hline No & 32 (37.6) & $33(33.8)$ & & $31(44.3)$ & $19(27.1)$ & \\
\hline Yes & $10(23.8)$ & $10(11.8)$ & & $10(14.3)$ & $10(14.3)$ & \\
\hline Death & & & $>.99$ & & & .724 \\
\hline No & $40(46.5)$ & 39 (45.3) & & 35 (49.3) & 27 (38.0) & \\
\hline Yes & $3(3.5)$ & $4(4.7)$ & & $6(8.5)$ & $3(4.2)$ & \\
\hline
\end{tabular}

Values are presented as number (\%).

PLK4, Polo-like kinase 4; CRT, chemoradiotherapy; TRG, tumor regression grade; KSP, The Korean Society for Pathologists; MSI, microsatellite instability; MSS, microsatellite stable; MSI-L, MSI-low; MSI-H, MSI-high.

.001), high pN category ( $\mathrm{p}=.026)$, microsatellite instability-high $(\mathrm{p}=.049)$, and high KSP-TRG $(\mathrm{p}<.001)$. There was no association between the Mandard-TRG and other parameters. The results mentioned above are summarized in Supplementary Table S1. High KSP-TRG correlated with high pT category $(\mathrm{p}<.001)$, high $\mathrm{pN}$ category $(\mathrm{p}=.011)$, and high Mandard-TRG $(\mathrm{p}<.001)$. There was no association between the KSP-TRG and other parameters. The results mentioned above are summarized in Sup- plementary Table S2.

Conversely, there was no significant association between DFS and high nuclear expression of PLK4 (p= .493) (Fig. 2A) or high cytoplasmic expression of PLK4 (p = .677) (Fig. 2B) in the preCRT specimens. There was also no significant association between OS and high nuclear expression of PLK4 ( $\mathrm{p}=.965)$ (Fig. 2C) or high cytoplasmic expression of PLK4 (p = .434) (Fig. 2D) in the pre-CRT specimens. 
Table 3. Correlation of PLK4 expression of post-CRT specimen with clinicopathological parameters in 102 cases of rectal cancer patients

\begin{tabular}{|c|c|c|c|c|c|c|}
\hline & \multicolumn{3}{|c|}{ Nuclear stain of PLK4 in post-CRT specimen } & \multicolumn{3}{|c|}{ Cytoplasmic stain of PLK4 in post-CRT specimen } \\
\hline & $\begin{array}{c}\text { Low expression } \\
\text { (H-score } \leq 160)\end{array}$ & $\begin{array}{c}\text { High expression } \\
(\mathrm{H} \text {-score }>160)\end{array}$ & $p$-value & $\begin{array}{c}\text { Low expression } \\
\text { (H-score } \leq 155)\end{array}$ & $\begin{array}{c}\text { High expression } \\
(H \text {-score }>155)\end{array}$ & $p$-value \\
\hline Age (yr) & & & .252 & & & .897 \\
\hline$<65$ & $17(25.4)$ & $18(26.9)$ & & $28(41.8)$ & $7(10.4)$ & \\
\hline$\geq 65$ & $20(29.9)$ & $12(17.9)$ & & $26(38.8)$ & $6(9.0)$ & \\
\hline Sex & & & .015 & & & .526 \\
\hline Male & $30(44.8)$ & $16(23.9)$ & & $38(56.7)$ & $8(11.9)$ & \\
\hline Female & $7(10.4)$ & $14(20.9)$ & & $16(23.9)$ & $5(7.5)$ & \\
\hline Differentiation & & & .251 & & & .332 \\
\hline Well & 0 & $2(3.1)$ & & $1(1.6)$ & $1(1.6)$ & \\
\hline Moderate & $34(53.1)$ & $24(37.5)$ & & $47(73.4)$ & $11(17.2)$ & \\
\hline Poorly & $2(3.1)$ & $2(3.1)$ & & $4(6.3)$ & 0 & \\
\hline Lymphovascular invasion & & & .849 & & & .318 \\
\hline Absent & $26(39.4)$ & $21(31.8)$ & & $36(54.5)$ & $11(16.7)$ & \\
\hline Present & $11(16.7)$ & $8(12.1)$ & & $17(25.8)$ & $2(3.0)$ & \\
\hline pT category & & & .057 & & & .317 \\
\hline $\mathrm{Tx}-\mathrm{T} 2$ & $8(11.9)$ & $13(19.4)$ & & $15(22.4)$ & $6(9.0)$ & \\
\hline T3-T4 & $29(43.3)$ & $17(25.4)$ & & 39 (58.2) & $7(10.4)$ & \\
\hline pN category & & & .154 & & & .753 \\
\hline NO & $26(38.8)$ & $16(23.9)$ & & 33 (49.3) & $9(13.4)$ & \\
\hline $\mathrm{N} 1-\mathrm{N} 2$ & $11(16.4)$ & $14(20.9)$ & & $21(31.3)$ & $4(6.0)$ & \\
\hline pM category & & & $>.99$ & & & .197 \\
\hline MO & $36(54.5)$ & $29(43.9)$ & & $53(80.3)$ & $12(18.2)$ & \\
\hline M1 & $1(1.5)$ & 0 & & 0 & $1(1.5)$ & \\
\hline TRG of Mandard & & & .918 & & & .279 \\
\hline TRG1, 2 & $2(3.0)$ & $1(1.5)$ & & $3(4.5)$ & 0 & \\
\hline TRG3 & $16(23.9)$ & $13(19.4)$ & & $21(31.3)$ & $8(11.9)$ & \\
\hline TRG4, 5 & $19(28.4)$ & $16(23.9)$ & & $30(44.8)$ & $5(7.5)$ & \\
\hline TRG of KSP & & & $>.99$ & & & $>.99$ \\
\hline TRGO, 1 & $2(3.0)$ & $1(1.5)$ & & $3(4.5)$ & 0 & \\
\hline TRG2, 3 & $35(52.2)$ & $29(43.3)$ & & 51 (76.1) & $13(19.4)$ & \\
\hline MSI & & & $>.99$ & & & .574 \\
\hline MSS and MSI-L & $34(50.7)$ & $28(41.8)$ & & 49 (73.1) & $13(19.4)$ & \\
\hline MSI-H & $3(4.5)$ & $2(3.0)$ & & $5(7.5)$ & 0 & \\
\hline Recurrence & & & .671 & & & .314 \\
\hline No & $25(37.9)$ & $21(31.8)$ & & $35(53.0)$ & $11(16.7)$ & \\
\hline Yes & $12(18.2)$ & $8(12.1)$ & & $18(27.3)$ & $2(3.0)$ & \\
\hline Death & & & .035 & & & .677 \\
\hline No & 29 (43.3) & 29 (43.3) & & $46(68.7)$ & $12(17.9)$ & \\
\hline Yes & $8(11.9)$ & $1(1.5)$ & & $8(11.9)$ & $1(1.5)$ & \\
\hline
\end{tabular}

PLK4, Polo-like kinase 4; CRT, chemoradiotherapy; TRG, tumor regression grade; KSP, The Korean Society for Pathologists; MSI, microsatellite instability; MSS, microsatellite stable; MSI-L, MSI-low; MSI-H, MSI-high.

\section{Multivariate analysis for the KSP-TRG}

Multivariate analysis showed that $\mathrm{pT}$ category and nuclear and cytoplasmic PLK4 expression in the pre-CRT specimens were statistically associated with the KSP-TRG $(\mathrm{p}<.05)$. Other variables, including age, sex, pN category, and MSI status, were not associated with the KSP-TRG. In addition, pT category and nuclear and cytoplasmic PLK4 expression in the pre-CRT specimens were statistically independent of each other or other variables. The results mentioned above are summarized in Table 4 .

\section{DISCUSSION}

In this study, we first distinguished the expression of immunohistochemical staining of PLK4 in samples before and after CRT by its high and low expressions and classified them according to the clinical data, including the prognosis and histopathological features. We also found that the expression of PLK4 in the pre-CRT specimens was associated with both the MandardTRG and KSP-TRG. This association was found in both nuclear 

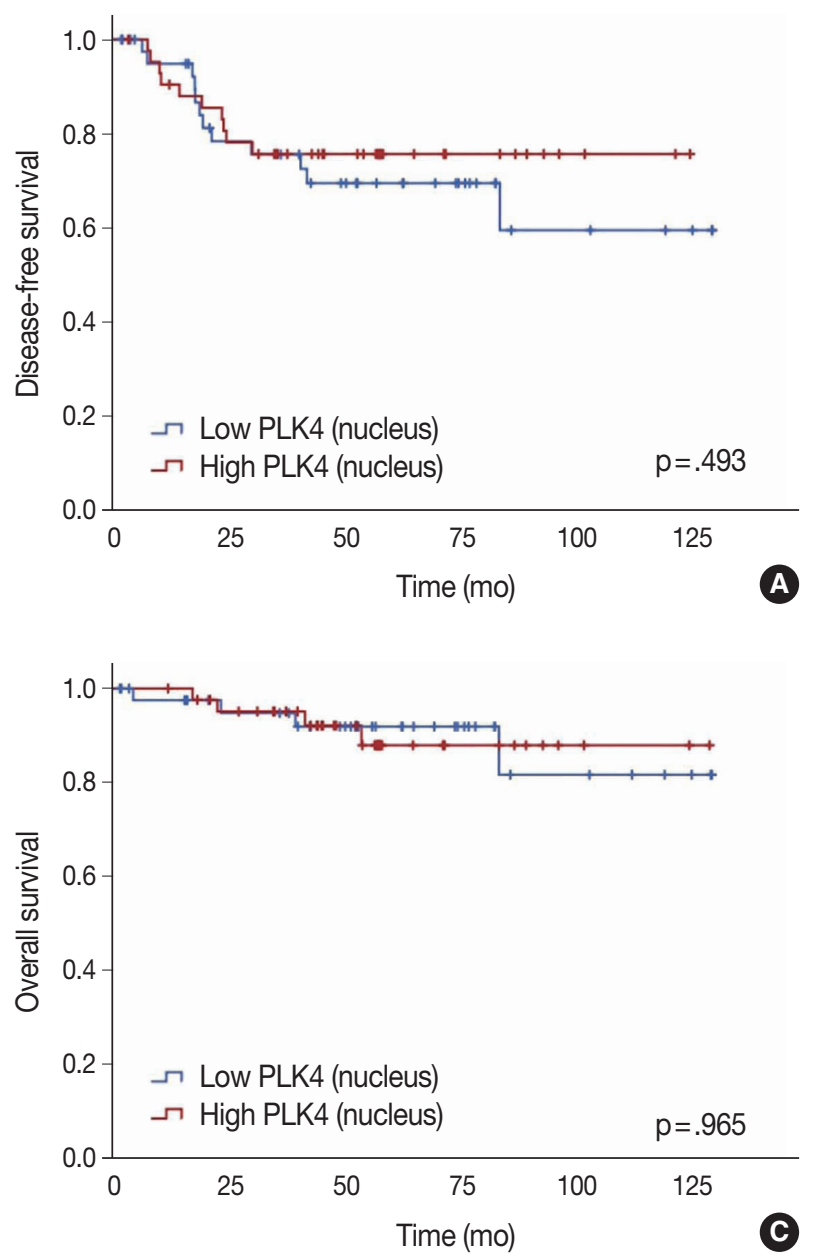
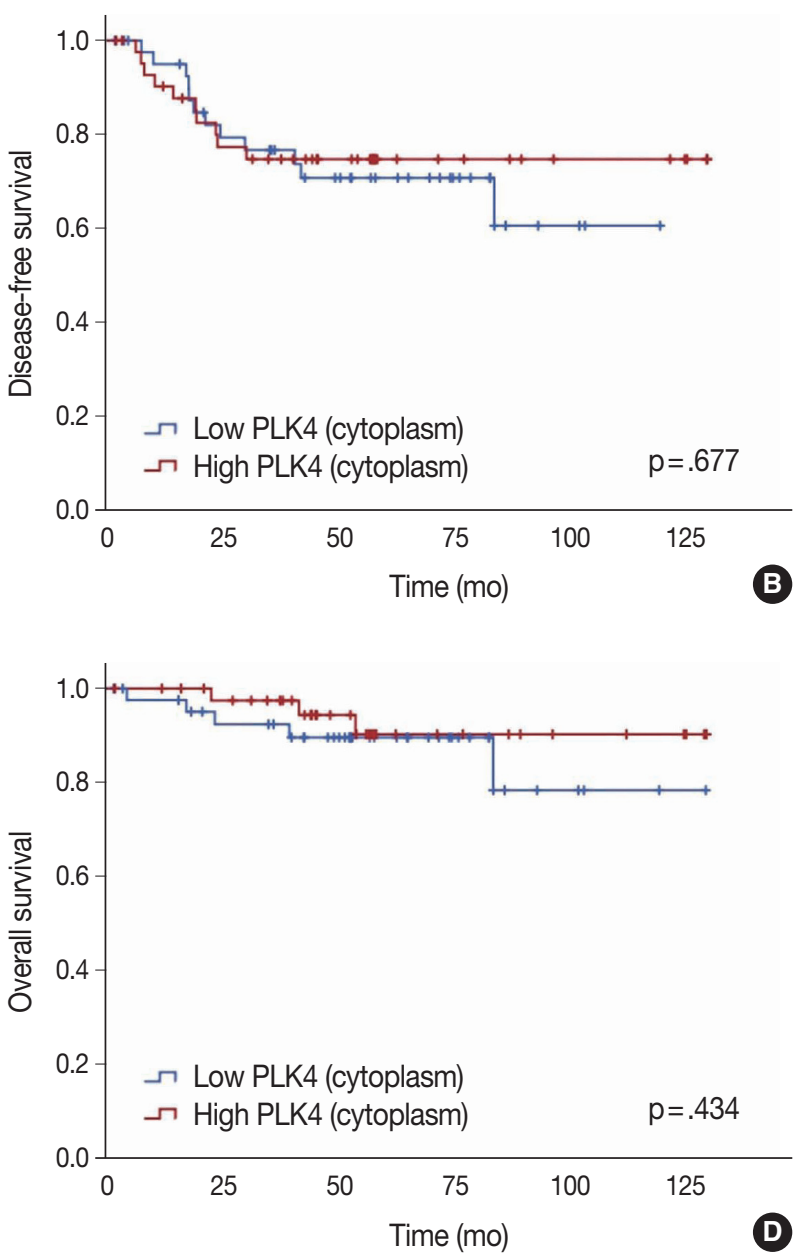

Fig. 2. (A) Kaplan-Meier curves of disease-free survival in high and low expression of nuclear expression of PLK4 of pre-CRT specimen. (B) Kaplan-Meier curves of disease-free survival in high and low expression of cytoplasmic expression of PLK4 of pre-CRT specimen. (C) Kaplan-Meier curves of overall survival in high and low nuclear expression of PLK4 of pre-CRT specimen. (D) Kaplan-Meier curves of overall survival in high and low expression of cytoplasmic expression of PLK4 of pre-CRT specimen. PLK4, Polo-like kinase 4; CRT, chemoradiotherapy.

Table 4. Multivariate analysis (ordinal logistic regression test) of TRG of KSP associated with clinicopathologic variables including nuclear and cytoplasmic PLK4 stain of pre-CRT specimen

\begin{tabular}{|c|c|c|c|}
\hline Variable & Estimate & p-value & 95\% Confidence interval \\
\hline \multicolumn{4}{|l|}{ Including nuclear PLK4 stain of pre-CRT specimen } \\
\hline Age & -0.685 & .113 & -1.532 to 0.161 \\
\hline Sex & -0.173 & .708 & -1.080 to 0.734 \\
\hline pT category & -1.403 & .003 & -2.338 to -0.469 \\
\hline pN category & -0.520 & .330 & -1.567 to 0.527 \\
\hline MSI state & -0.578 & .406 & -1.939 to 0.784 \\
\hline Nuclear PLK4 stain of pre-CRT specimen & -1.766 & $<.001$ & -2.667 to -0.866 \\
\hline \multicolumn{4}{|c|}{ Including cytoplasmic PLK4 stain of pre-CRT specimen } \\
\hline Age & -0.411 & .329 & -1.237 to 0.415 \\
\hline Sex & -0.351 & .444 & -1.250 to 0.548 \\
\hline pT category & -1.595 & .001 & -2.532 to -0.658 \\
\hline pN category & -0.513 & .335 & -1.558 to 0.531 \\
\hline MSI state & -0.444 & .530 & -1.829 to 0.940 \\
\hline Cytoplasmic PLK4 stain of pre-CRT specimen & -1.500 & .001 & -2.376 to -0.623 \\
\hline
\end{tabular}

TRG, tumor regression grade; KSP, The Korean Society for Pathologists; PLK4, Polo-like kinase 4; CRT, chemoradiotherapy; MSI, microsatellite instability. 
and cytoplasmic expression. Therefore, preoperative evaluation of PLK4 will be useful as one of the methods to predict the response of rectal cancer patients to CRT. Unfortunately, the association between PLK4 and prognosis was not revealed in this study and more robust studies are needed in the future to support this possibility.

Although radiation therapy before surgery reduces the local recurrence rate by more than $50 \%$ than surgery alone $[1]$ and treatment for rectal cancer has greatly evolved in recent years, patients are prone to recurrence and many eventually die from the disease. Therefore, the importance of the response to preoperative CRT in rectal cancer is very important.

In a study on the correlation between preoperative CRT and PLK4 expression, PLK4 enhanced chemoradiation resistance in glioblastoma multiforme (GBM), whereas PLK4 knockdown via lentiviral transfection significantly increased the chemoradiation sensitivity of GBM cells [17]. The authors stated that PLK4 was transcriptionally regulated depending on ATAD2 (ATPase family AAA domain containing 2), a gene involved in cell proliferation and metastasis, and that this regulation increased PLK4 radioresistance. In addition, PLK4 expression is known to be a negative predictor of response to taxane-based neoadjuvant chemotherapy [18]. The authors stated that the resistance to taxanebased chemotherapy appeared in patients with high PLK4 expression because PLK4 was involved as an upstream regulator of gamma-tubulin, which affected the treatment response to taxane. Therefore, the authors concluded that PLK 4 inhibitors enhanced the effect of chemotherapy. However, reports on the possibility of prognostic effects of PLK4 expression in rectal cancer are lacking.

There are several drawbacks to our study. The most significant drawback was the limited number of cases. Despite the small sample size that could undermine the power of the study, we found a significant difference, which should be confirmed by large-scale studies in the future. Additionally, the retrospective nature of the study was a limitation to its design. It would also be necessary to design a better-validated study with no missing factors. Future research with better power should be conducted to confirm the preliminary results obtained from this study.

We reported PLK4 expression and the prognosis of rectal cancer by dividing our study into pre-CRT and post-CRT groups. Based on our human tissue study results, PLK4 expression could be a potential predictor for the response of CRT. Therefore, the clinical development of PLK4 could be an effective therapeutic strategy for the management of advanced rectal cancer.

\section{Supplementary Information}

The Data Supplement is available with this article at https://doi.org/10.4132/ jptm.2021.10.07.

\section{Ethics Statement}

All procedures performed in the current study were approved by the Institutional Review Board (IRB) of the Keimyung University Dongsan Hospital (DSMC 2019-09-007-002) in accordance with the 1964 Helsinki declaration and its later amendments. Formal written informed consent was not required with a waiver by the IRB.

\section{Availability of Data and Material}

The datasets generated or analyzed during the study are available from the corresponding author on reasonable request.

\section{Code Availability}

Not applicable.

\section{ORCID}

Hyunseung Oh https://orcid.org/0000-0001-9440-189X Soon Gu Kim https://orcid.org/0000-0002-1436-8442 Sung Uk Bae https://orcid.org/0000-0002-7876-4196 Sang Jun Byun https://orcid.org/0000-0002-6115-2804 Shin Kim https://orcid.org/0000-0002-1099-5027 Jae-Ho Lee https://orcid.org/0000-0002-5562-0720 Ilseon Hwang https://orcid.org/0000-0002-6122-4417 Sun Young Kwon https://orcid.org/0000-0002-8410-0185 Hye Won Lee https://orcid.org/0000-0001-8540-524X

\section{Author Contributions}

Conceptualization: HWL. Methodology: HO, IH, SGK, HWL. Data curation: SUB, SJB, SK, JHL. Formal analysis: HO, IH, SGK. Supervision: SYK. Visualization: HO, SGK. Writing_original draft: HO, HWL. Writing-review \& editing: HO, IH, SYK, HWL. Approval of final manuscript: all authors.

\section{Conflicts of Interest}

The authors declare that they have no potential conflicts of interest.

\section{Funding Statement}

This work was supported by the National Research Foundation of Korea (NRF) grant funded by the Korea government (MSIT) (No. 2017R1C1B 5077166).

\section{References}

1. Camma C, Giunta M, Fiorica F, Pagliaro L, Craxi A, Cottone M. Preoperative radiotherapy for resectable rectal cancer: a meta-analysis. JAMA 2000; 284: 1008-15.

2. Bettencourt-Dias M, Rodrigues-Martins A, Carpenter L, et al. SAK/PLK4 is required for centriole duplication and flagella development. Curr Biol 2005; 15: 2199-207.

3. Habedanck R, Stierhof YD, Wilkinson CJ, Nigg EA. The Polo kinase Plk4 functions in centriole duplication. Nat Cell Biol 2005; 7: 1140-6.

4. Sillibourne JE, Tack F, Vloemans N, et al. Autophosphorylation of polo-like kinase 4 and its role in centriole duplication. Mol Biol Cell 2010; 21: 547-61.

5. Rodrigues-Martins A, Riparbelli M, Callaini G, Glover DM, Bet- 
tencourt-Dias M. Revisiting the role of the mother centriole in centriole biogenesis. Science 2007; 316: 1046-50.

6. Macmillan JC, Hudson JW, Bull S, Dennis JW, Swallow CJ. Comparative expression of the mitotic regulators SAK and PLK in colorectal cancer. Ann Surg Oncol 2001; 8: 729-40.

7. Mason JM, Lin DC, Wei X, et al. Functional characterization of CFI-400945, a Polo-like kinase 4 inhibitor, as a potential anticancer agent. Cancer Cell 2014; 26: 163-76.

8. Denu RA, Zasadil LM, Kanugh C, Laffin J, Weaver BA, Burkard ME. Centrosome amplification induces high grade features and is prognostic of worse outcomes in breast cancer. BMC Cancer 2016; 16: 47.

9. Coelho PA, Bury L, Shahbazi MN, et al. Over-expression of Plk4 induces centrosome amplification, loss of primary cilia and associated tissue hyperplasia in the mouse. Open Biol 2015; 5: 150209.

10. Sercin O, Larsimont JC, Karambelas AE, et al. Transient PLK4 overexpression accelerates tumorigenesis in p53-deficient epidermis. Nat Cell Biol 2016; 18: 100-10.

11. Ko MA, Rosario CO, Hudson JW, et al. Plk4 haploinsufficiency causes mitotic infidelity and carcinogenesis. Nat Genet 2005; 37: 883-8.

12. Rosario CO, Ko MA, Haffani YZ, et al. Plk4 is required for cytoki- nesis and maintenance of chromosomal stability. Proc Natl Acad Sci U S A 2010; 107: 6888-93.

13. Kazazian $\mathrm{K}$, Go C, Wu H, et al. Plk4 promotes cancer invasion and metastasis through Arp2/3 complex regulation of the actin cytoskeleton. Cancer Res 2017; 77: 434-47.

14. Mandard AM, Dalibard F, Mandard JC, et al. Pathologic assessment of tumor regression after preoperative chemoradiotherapy of esophageal carcinoma: clinicopathologic correlations. Cancer 1994; 73: 2680-6.

15. Kim BH, Kim JM, Kang GH, et al. Standardized pathology report for colorectal cancer, 2nd edition. J Pathol Transl Med 2020; 54: 1-19.

16. McCarty KS Jr, Miller LS, Cox EB, Konrath J, McCarty KS Sr. Estrogen receptor analyses. Correlation of biochemical and immunohistochemical methods using monoclonal antireceptor antibodies. Arch Pathol Lab Med 1985; 109: 716-21.

17. Wang J, Zuo J, Wang M, et al. Pololike kinase 4 promotes tumorigenesis and induces resistance to radiotherapy in glioblastoma. Oncol Rep 2019; 41: 2159-67.

18. Li Z, Dai K, Wang C, et al. Expression of polo-like kinase 4 (PLK4) in breast cancer and its response to taxane-based neoadjuvant chemotherapy. J Cancer 2016; 7: 1125-32. 